\title{
Improvising Dynamic Cloud Resource Allocation to Optimise QoS and Cost Effectiveness
}

\author{
Gudur Vamsi Krishna, K. F. Bharati
}

\begin{abstract}
Cloud computing offers streamlined instruments for outstanding business efficiency processes. Cloud distributors typically give two distinct forms of usage plans: Reserved as well as On-demand. Restricted policies provide inexpensive long-term contracting services, while order contracts were very expensive and ready for brief rather than long longer periods. In order to satisfy current customer demands with equal rates, cloud resources must be delivered wisely. Many current works depend mainly on low-cost resource-reserved strategies, which may be under-provisioning and over-provisioning rather than costly ondemand solutions. Since unfairness can cause enormous high availability costs and cloud demand variability in the distribution of cloud resources, resource allocation has become an extremely challenging issue. The hybrid approach to allocating cloud services according to complex customer orders is suggested in that article. The strategy was constructed as a two-step mechanism consisting of accommodation stages and then a versatile structure. In this way, by constructing each step primarily as an optimization problem, we minimize the total cost of implementation, thereby preserving service quality. By modeling client prerequisites as probability distributions are disseminated owing to the dubious presence of cloud requests, we set up a stochastic Optimization-based approach. Using various approaches, our technique is applied, and the results demonstrate its effectiveness when assigning individual cloud resources.
\end{abstract}

Keywords - Cloud computing, Resource allocation, Demand

\section{INTRODUCTION:}

Cloud networking has become a common model of connectivity that provides Internet services[1],[2]. In order to deliver their applications, cloud computing allows network service providers to reduce the expense of hardware resources. In addition, the ease of resource creation and the quick response time was the important aspect that expanded awareness of cloud computing[3],[4]. Infrastructure-as-aservice cloud (IaaS) was the subject of this study, where cloud providers include infrastructure services such as networks, servers, databases, etc. Actually, with internet service providers with distinct resource-based pricing schemes[5]-[7], cloud networks suggest different sort of IaaS resource delivery services, Reserved and On-demand proposals. Using reserved plans, Web service providers could collect market prices and expect to be paid once with the term of the agreement (e.g. one-year agreement instead of a three-year Amazon EC2 agreement)[8]. By the means of on-demand plans, cloud providers deliver extra dynamic bandwidth marketing strategies.

Manuscript received on February 25, 2021.

Revised Manuscript received on February 28, 2021.

Manuscript published on February 28, 2021

* Correspondence Author

Gudur Vamsi Krishna*, M. Tech, Department of CSE, JNTUACE, Anantapur, India

Dr. K. F. Bharati, Assistant Professor, Department of CSE, Anantapur, India

(C) The Authors. Published by Blue Eyes Intelligence Engineering and Sciences Publication (BEIESP). This is an open access article under the CC BY-NC-ND license (http://creativecommons.org/licenses/by-nc-nd/4.0/)
On-demand proposals pay for controlled cloud Pay-as-yougo utility distributors and encourage every time depending on user requirements to start rather than finish examples without paying the particular penalty. Comparing the cost of resources per unit for a period, Nevertheless with-order resources are much more expensive than those allocated with the local intention, web services provide cases in advance with future economic deals. Sustainable investment will be a tricky task for only the quiet situations due to uncertainty of the complexities in the quiet plans. The funds purchased may not have been equipped to satisfy the criteria, leading to undersupply. In the face of economic uncertainty, a failure to achieve Quality of Service (QoS) could be a critical problem for both cloud and network service providers[9]. On the other hand, though, too much provisioning can arise when for the most part, financially stable supplies are often unrealistic for the management of actual emerging needs[1], resulting in needless costs. Cloud resource management has recently been defined in a few studies, but cloud web service providers have become required to handle data delivery responsibilities[9]-[11]. As a single-step algorithm, many current cloud resource allocation methods design resource allocations [12]-[15]. Under such operations, the writers ignore market ambiguity while suggesting probabilistic market values. Therefore, the dynamic existence of cloud-based systems is not known. In order to solve Demand's complexities, multiple dynamic resource distribution algorithms are built-in[16]-[19]. These techniques are a way flexible and spread cloud services intensely to reduce the expense of energy distribution. These operations do not optimize the cost savings by the reserved contracts which are provided by cloud vendors. They will then fail to come up with financial responses. The hybrid approach to assigning specialized computing resources to create cloud-based mobile applications is proposed in the studies. By taking the advantage of the allocated and withorder resources, but also of the scalable strategy, which minimizes the cost of production and ensures QoS through output uncertainty. The key results of the entire article are outlined in the following points: Proposing Dynamic Cloud Resource Allocation (DCRA) approach that discusses simple information over two allocated but also dynamic delivery measures, applying a probabilistic system of improvement as free variables for design customer orders, as well as achieving an increase of 10 percent overall price. In Amazon Web Services [7] and Microsoft Azure cloud[6], including cloud distributors using two distinct benchmark workloads, a proposed DCRA mechanism was tested. The results reveal that the proposed DCRA approach recognizes alternatives under uncertainties in the requirements of the customers minimize the total cost of implementation. 


\section{Improvising Dynamic Cloud Resource Allocation to Optimise QoS and Cost Effectiveness}

\section{RELATED WORK}

The issue of cloud asset provisioning has carried specialists' consideration regarding giving asset portion calculations and methods in the previous few years. Most asset allotment works to frame the issue like single-stage calculation that takes into account of assets with reserved cloud suppliers' plans. To adapt to this problem, on-request asset provisioning strategies are proposed to allow assets as indicated by the dynamic cloud request.

\subsection{Deterministic Resource Provisioning}

Jiao et al. presented a cost model for the implementation of the online social network[13]. The analysis was planned to optimize the monitoring price of accessing cloud resources when running QoS, such as latency in access. The event that the area of the client is changed, the first arrangement will no longer meet the QoS specification. Expenditure was optimized using such a heuristic algorithm; however the answer could only be a local optimum.Similarly, a multiobjective framework[21] is proposed to reduce total deployment costs and to maximize QoS performance at the same time, while being opposing goals. This approach requires a single stage optimization that only takes into account the cloud resources assigned to the application. In [12], Imai et al. suggested the use of agnostic performance modelling for a large application class to reduce costs. In order to increase the accuracy of the forecast, a probabilistic predictive capability is applied to the model to predict the performance of the application. In order to comply with a Service Level Agreement (SLA), such as a latency violation, the maximum throughput of users is considered to establish a model. All of these works suggest deterministic methods to model various resource allocation concerns that can contribute to under-and over-provisioning. In this article, a dynamic cloud asset assignment algorithm is proposed to relegate assets concurring to complex customer necessities.

\subsection{Dynamic Resource Provisioning}

In [12],An application-agnostic algorithm to minimize costs for a wide variety of applications has been evoked. An Optimum Cloud Resource Provisioning (OCRP) algorithm is proposed to minimize the overall cost of delivering services over a specific time frame. This algorithm has been broken down into three modules[13]. In the event that the resources obtained cannot fulfill the specifications, further resources will be purchased at the on-demand level. Since the ambiguity model is discreet, decomposition strategy is utilized within

the OCRP algorithm to unravel the current sub-problems and conserve runtime, the accuracy of the algorithm results is affected. Chaisiri et al. lowered the cost of delivering services through the Robust Cloud Resource Provisioning (RCRP) algorithm[9]. Proposed RCRP allows for three step cloud providers. The study as a whole uses three sources of volatility: uncertainty in the availability of demand and costs as cloud providers. Allocations are made during the first phase where the applicant is given a certain sum of money. In the expenditure process, the usage of available capital has been seen to consider which facilities are under-or oversupplied. Where allocated resources have not been able to meet the targets, additional resources must be delivered in on-demand facilities during the on-demand process. The Outcomes demonstrate that the RCRP algorithm renders low on-demand costs compared to the contemporary works.
While a systematic change is used in the proposed RCRP process, a fatalistic approach is required, resulting in a high cost. Moreover, such a technique does not differentiate between the types of computational and network storage tools from simulation issues. Throughout [22], Ran et al. proposed a cost-effective, high-availability solution to economic productivity across the Internet network. The purpose of this study appears to be to reduce the purchasing price of capital and to maximize the gains by taking into account the complexity of both demand and cost. The proposed methodology involves two stages of stochastic programming. Second, the equivalent concept of determinism is expressed to minimize the cost of acquiring Virtual Machines (V.M.). The profit was maximized in the second stage by the capital storage facility. Yu et al. proposed a synthetic cloud distribution approach to reduce the expense of lodging by bandwidth[23]. In addition to understanding Demand's uncertainty, a synthetic deterministic ensemble will be proposed to ensure that renters' expectations for bandwidth were indeed met. The goal is to reduce the maximum usage of resources in [20]. The probabilistic linear optimization approach for cloud resource requirements has also been explored in this work. Compared to OCRP and RCRP algorithms that use pessimistic methods using rigorous worst-case optimizationIn order to discover a balanced arrangement and dodge superfluous costs due to negative arrangements, We propose a stochastic optimization approach. At last, not at all like the work proposed in[20]that considers dubious framework vulnerability from a cloud distributor's perception, our proposed DCRA algorithm addresses the issue of complex cloud asset allotment from a provider's aspect.

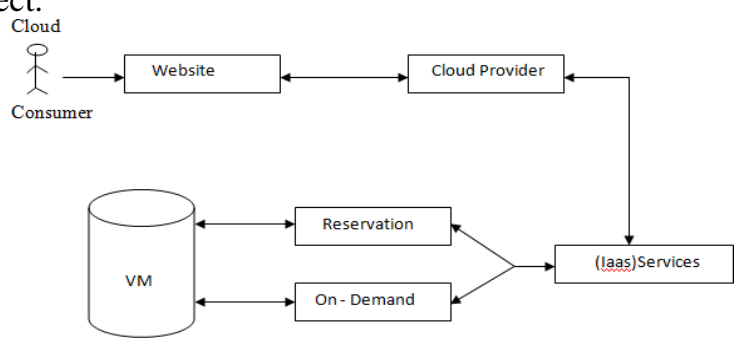

Figure1: Work Flow

\section{METHODOLOGY}

\section{Dynamic Allocation Of Cloud Resources}

Mostly the Cloud Vendors rely on long durated Contracts(on a yearly stand).On-demand services, on the other hand, are obtained for some arbitrary time frame, normally in hours. Reserved services are known to have a single pay whereas on-demand resources are paid during the time period they are used. The price of these resources depends based on the length of the subscription, the long term or the short term. As shown, the Amazon pricing model[7] and the GoGrid[24] pricing model are seen in Table 1. For instance, in nearly all cloud vendors, Amazon and Azure, assets retained are held safe by long-distance contracts. In the other hand, on-demand assets are procured for every self-assertive timeframe, and on a routine basis, assets are paid for the timeframe they are used.

Blue Eyes Intelligence Engineering

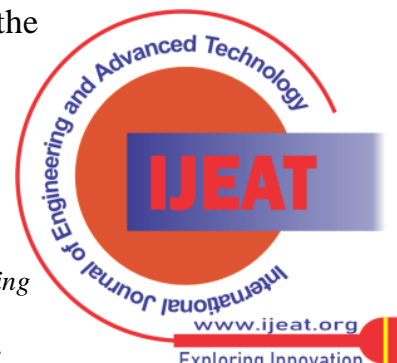


They address the complex needs of V.M.s with different asset configurations. The cost of these properties varies based on the duration of the membership, the length of the membership, or the current period. As customer requests for software are not deterministic, web-based expert organisations purchase cost-effectiveness through a mix of holdings and requesting cloud administration.

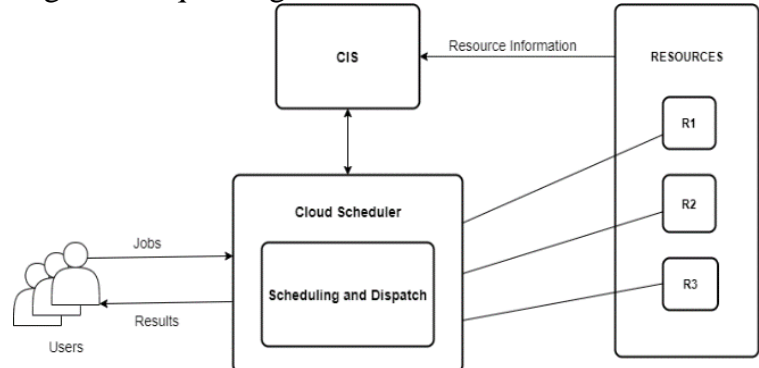

Figure 2: Web service deployment Cloud

\section{TABLE 1 Parameters based on AWS and Azure on Resources}

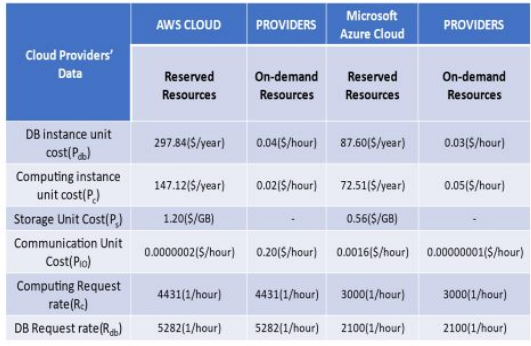

However, the current cloud resources component works; it neglects to consider both the stored and the on-demand forms of assets under or over provisioning. Proposed Dynamic Cloud Capital Sharing Restricts Cumulative Spending of Keep and On Demand Administrations. It prompts a response that keeps the web application going under changes of interest. Cloud vendors supply various types of V.M.s with different resource settings in order to satisfy different consumer specifications. Monopolies such as Amazon and Azure, and GoGrid, fixed resource rates on the basis of the two resource allocation plans: the reserved package and the on-demand plan. As a result, two distinct costs are correlated with V.M.s in cloud models. Distinct resource specifications and QoS standards reckon to help the application of service providers.

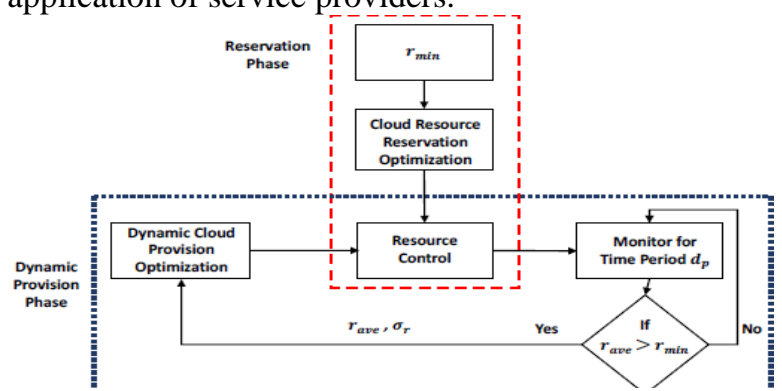

Figure 3:Dynamic Cloud Resource Allocation

Here ' $r$ ' implies the frequency of requests, The $d_{p}$ indicates Duration for Dynamic Cloud Provisioning.

\section{DYNAMIC CLOUD RESOURCE ALLOCATION ALGORITHM}

This segment proposes a two-phase calculation that minimizes the taken a toll of web server usage. Within the first stage (alluded to as the Reservation stage), administrations from the Reservation Plan are saved for web application execution to fulfill the minimum QoS details. In the second stage (alluded to as the complex arrangement stage), non-deterministic client determinations are displayed as arbitrary factors.

\subsection{DCRA Flowchart Overview}

The phases of allocation, namely the phase of reservation and the phase of complex provision, are presented in Fig. 2. Cloud vendors sell a particular sort of services as reserved to online service providers in the form of long durated contracts at cheaper costs. For eg, Amazon's EC2[7] reserved package is available in a year or multiples of it, while Go Grid[24] provides yearly and monthly contracts.

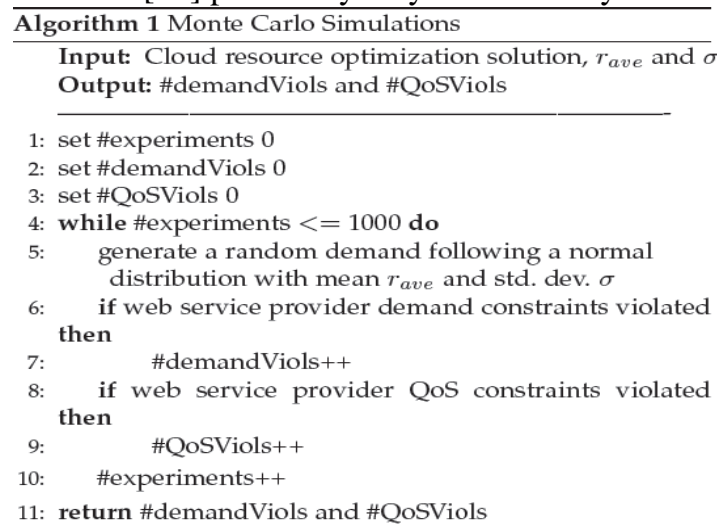

The algorithm sets the required capital to fulfill the minimum customer request rmin for the services of the reservation process. Note that rmin is provided as input by the web service provider only once at the start of the implementation service period and is never measured or updated later. These Conclusions are drawn from the employee based data set of allocation and usage of the resources. Using proposed DCRA we will minimize the expense of deploying web apps, even with demand volatility. With the optimization of Cloud Resource Reservation.

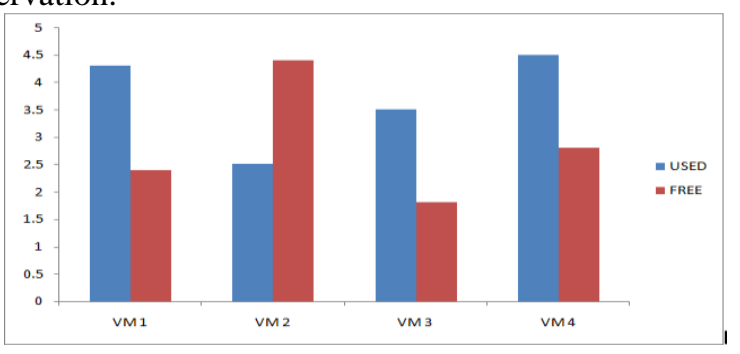

Fig : Resource allocation and their usage statistics cost of reservation is reduced as the least expected requirements are considered to be identified as a-priori.

\section{EXPERIMENTAL SETUP}

Any cloud infrastructure environment can be used in our assessment. However we are using two common cloud vendors, AWS and Azure to obtain cloud tools for deployment of Web applications. The proposed DCRA algorithm and experimental algorithms are built using the $\mathrm{C}++$. Mosek 6.0[27] solver is utilized to overcome the optimization issues defined as the proposed technique.

Published By:

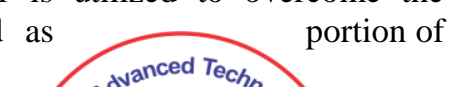

Blue Eyes Intelligence Engineering and Sciences Publication (C) Copyright: All rights reserved. 


\section{Improvising Dynamic Cloud Resource Allocation to Optimise QoS and Cost Effectiveness}

The simulation set-up is being developed in the $\mathrm{C}++$ environment. The rate of arrival of customer demand is modeled on the logically dependent queuing model set out in [25]. The output data of cloud provider providers[6],[7] were used to model cloud resource behaviors. Monte Carlo models are also used to test solution to problems of unknown nature[17],[22],[26]. The input parameters of the Monte Carlo Re-enactments calculation are the DCRA or QCost cloud asset optimization approach . Since client demands for a web application are arbitrary factors taking after typical disseminations, the cruel and standard deviation of the application are regularly considered.

\section{CONCLUSION}

A cost-effective hybrid provisioning algorithm for serverbased computer program applications within the cloud setting is proposed that centers on maximizing the by and large fetched of provisioning whereas taking under consideration the instability of shopper prerequisites. The DCRA which is proposed is divided into two phases, The former is Reservation and the latter Dynamic Provisioning phase. In order to test DCRA's performance, Reenactments have been conducted for different workload scenarios. The discoveries appear that the proposed procedure can give effective and cost-effective arrangements employing a complex blend of distributed and on-demand administrations to send cloud-based applications. In comparison, the planned DCRA is an autonomous cloud service that can be used by major cloud ditributors such as AWS[7], Azure[6] and Go Grid[24]. The proposed work extends DCRA to automatically distribute services to various cloud providers' applications in order to achieve minimal costs and greater redundancy. In future work,Priority scheduling techniques may be used during resource allocation from the clients.

\section{REFERENCES}

1. Mireslami, S., Rakai, L., Wang, M., \& Far, B. H. (2019).” Dynamic Cloud Resource Allocation Considering Demand Uncertainty”. IEEE Transactions on Cloud Computing, 1-1.

2. M.Anastasopoulos, A.Tzanakaki, and D. Simeonidou, "Stochastic energy-efficient cloud service Provisioning deploying renewable energy sources," IEEE Journal on Selected Areas in Communications, vol. 34, no. 12, pp. 3927-3940, Dec 2016.

3. A. Johannes, N. Borhan, C. Liu, R. Ranjan, and J. Chen, "A user demand uncertainty based approach for cloud resource management," in 2013 IEEE 16th International Conference on Computational Science and Engineering, Dec 2013, pp. 566-571.

4. S. Hosseinalipour and H. Dai, "Options-based sequential auctions for dynamic cloud resource allocation," in 2017 IEEE International Conference on Communications (ICC), May 2017, pp. 1-6.

5. Rackspace. https://www.rackspace.com

6. Microsoft Azure. http://azure.microsoft.com/

7. Amazon web services. http://aws.amazon.com/.

8. AWS Amazon EC2. https://aws.amazon.com/ec2/pricing/on-demand/.

9. S.Chaisiri, B.S.Lee, and D. Niyat, "Robust cloud resource provisioning for cloud computing environments," in 2010 IEEE Applications (SOCA), Dec 2010, pp. 1-8.

10. N.Sfika, A.Korfiati, C.Alexakos, S. Likothanassis, K.Daloukas, \& P. Tsompanopoulou, "Dynamic cloud resources allocation on multidomain/multiphysics problems," in 2015 3rd International Conference on Future IoT and Cloud, Aug 2015, pp. 31-37.

11. R.I.Meneguette,A.Boukerche, A.H.M.Pimenta, \& M.Meneguette, "A resource allocation scheme based on semi-Markov decision process for dynamic vehicular clouds," in 2017 IEEE International Conference on Communications (ICC), May 2017, pp.1-6.

12. S.Imai, S.Patterson, \& C.A.Varela, "Cost-efficient elastic stream processing using application-agnostic performance prediction," in International Conference on Service-Oriented Computing and

2016 16th IEEE/ACM International Symposium on Cluster, Cloud and Grid Computing (CCGrid), May 2016, pp. 604-607.

13. L. Jiao, J. Li, T. Xu, W. Du, and X. Fu, "Optimizing cost for online social networks on geo-distributed clouds," IEEE/ACM Transactions on Networking, vol. 24, no. 1, pp. 99-112, Feb 2016.

14. H. Goudarzi, M. Ghasemazar, and M. Pedram, "SLAbased optimization of power and migration cost in cloud computing," in the 12th IEEE/ACM International Symposium on Cluster, Cloud and Grid Computing (CCGrid), May 2012, pp. 172-179.

15. W. K. Tan, D. M. Divakaran, and M. Gurusamy, "Uniform price auction for allocation of dynamic cloud bandwidth," in 2014 IEEE International Conference on Communications (ICC), June 2014, pp. 2944-2949.

16. J. Chase and D. Niyato, "Joint optimization of resource provisioning in cloud computing," IEEE Transactions on Services Computing, vol. PP, no. 99, pp. 1-1, 2015.

17. S. Chaisiri, B. S. Lee, and D. Niyato, "Optimization of resource provisioning cost in cloud computing," IEEE Transactions on Services Computing, vol. 5, pp. 164-177, April 2012.

18. J. N. Khasnabish, M. F. Mithani, and S. Rao, "Tiercentric resource allocation in multi-tier cloud systems," IEEE Transactions on Cloud Computing, vol. 5, no. 3, pp. 576- 589, July 2017.

19. B.Neethu and K.R.R.Babu, "Dynamic resource allocation in market oriented cloud using auction method," in 2016 International Conference on Micro-Electronics and Telecommunication Engineering (ICMETE), Sept 2016, pp. 145-150.

20. M.Anastasopoulos, A. Tzanakaki, and D. Simeonidou, "Stochastic energy efficient cloud service provisioning deploying renewable energy sources," IEEE Journal on Selected Areas in Communications,vol. 34, no. 12, pp. 3927-3940, Dec 2016.

21. S. Mireslami, L. Rakai, B. H. Far, and M. Wang, "Simultaneous cost and qos optimization for cloud resource allocation," IEEE Transactions on Network and Service Management, vol. 14, no. 3 , pp.676-689, Sept 2017.

22. Y. Ran, B. Yang, W. Cai, H. Xi, and J. Yang, "Cost-efficient provisioning strategy for multiple services in distributed clouds,"in 2016 International Conference on Cloud Computing Research and Innovations (ICCCRI), May 2016, pp. 1-8.

23. L. Yu and H. Shen, "Bandwidth guarantee under demand uncertainty in multi-tenant clouds," in 2014 IEEE 34th International Conference on Distributed Computing Systems, June 2014, pp. 258-267.

24. GoGrid. https://www.datapipe.com/gogrid/

25. X. Nan, Y. He, and L. Guan, "Optimal Resource Allocation for Multimedia Cloud Based on Queuing Model," in the 13th IEEE International Workshop on Multimedia Signal Processing (MMSP), Hangzhou, China, October 17-19 2011, pp. 1-6.

26. A. Dastjerdi, S. Garg, and R. Buyya, "QoS-aware deployment of network of virtual appliances across multiple clouds," in IEEE International Conference on Cloud Computing Technology and Science(CloudCom), November 2011, pp. 415-423.

27. Mosek 6.0. http://www.mosek.com.

\section{AUTHORS PROFILE}

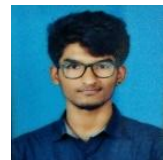

Gudur Vamsi Krishna received B.Tech in Compute Science and Engineering from Jawaharlal Nehru TechnologicalUniversity, Pulivendula in 2018.Currently pursuing M.tech in Computer Science and Engineering under Specialisation in Computer Science(CS) from JNTUA College of Engineering,Ananthapuramu,Andhrapradesh,India.His areas of interests include Cloud Computing,Data base Systems.

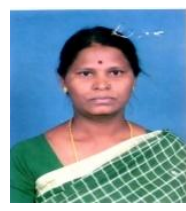

Dr.K.F.Bharati is an expert in area of Data Mining,Cloud Computing and has taught subjects in this area for more than fifteen years in reputed colleges and Universities.She received Ph.D from JNTUA University,Ananthapuramu.Currently working asAssistant professor in Department of Computer Science and Engineering,JNTUA College of Engineering,Ananthapuramu,AndhraPradesh,India.Her areas of interest include Data Mining and Cloud Computing.

\section{Published By:}

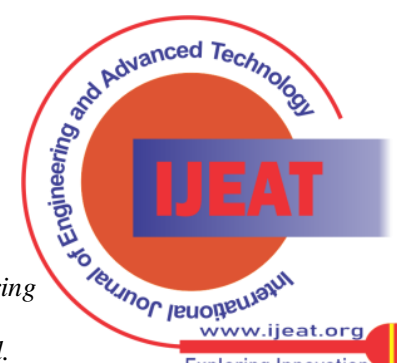

